04

\title{
Исследования воздействия пинчевых плазмоидов в периодическом разряде в потоке жидкости на окружающую среду
}

\author{
(C) А.В. Нестерович \\ Национальный исследовательский ядерный университет „МИФИ“, \\ 115409 Москва, Россия \\ e-mail: frontlines.07@gmail.com
}

Поступило в Редакцию 14 декабря 2018 г.

В окончательной редакции 14 декабря 2018 г.

Принято к публикации18 марта 2019 г.

Периодический разряд в потоке жидкости является одним из эффективных средств модифицирования поверхности металлических изделий с целью повышения их износоустойчивости, твердости и коррозийной стойкости. Изучение свойств разряда с целью определения предельных возможностей данного метода и оптимизации технологических режимов обнаружило ряд физических явлений, которые ранее не были известны. Учет данных факторов необходим при определении мер безопасности при реализации промышленных технологий. Новым элементом в рассмотрении является учет обнаруженных аномальных проявлений новообразований типа шариков, треков и нитей в окружающей среде.

Ключевые слова: разряд, поверхность, плазмоид, свечение, магнитные заряды, гравитационная модель.

DOI: $10.21883 /$ JTF.2019.09.48059.432-18

\section{Введение}

Одним из перспективных методов, реализующих воздействие электрических разрядов на материалы, является метод, основанный на формировании периодического высоковольтного разряда в потоке жидкости (ПРПЖ) [1-4]. Применение струи жидкости позволяет реализовать одновременно функции токоподводящего электрода и коммутатора, с помощью которого формируется последовательность импульсов электрического разряда длительностью порядка нескольких наносекунд и более. В условиях быстропротекающего электрического разряда в приэлектродном пространстве и на поверхности плоского электрода создаются условия экстремальных состояний (высокая напряженность электрического и магнитного полей, ударно-волновое воздействие, избыток электронной составляющей, наличие воды, ионов $\mathrm{H}^{+}, \mathrm{O}^{+}, \mathrm{OH}^{+}$и др.). Данный метод уже имеет технологическое применение в процессах упрочнения и повышения износоустойчивости конструкционных материалов [5].

\section{Методика эксперимента}

Суть метода ПРПЖ заключается в том, что при подаче напряжения на натекатель от источника питания с падающей вольт-амперной характеристикой возникает квазипериодический сильноточный разряд по поверхности струи жидкости. При этом поток жидкости обрывается за счет воздействия на него ударных волн, возникающих при разряде, т. е. коммутация тока осуществляется автоматически.
Исследование электрических и оптических характеристик разряда выполнено двумя методами: методом получения двухмерного изображения и методом получения трехмерного (стереоизображения) с использованием высокоскоростных камер. На первом этапе работ применена скоростная киносъемочная камера СКС-1М со скоростью съемки от 150 до 4000 кадров в секунду. Для получения стереоизображения съемка проводилась двумя синхронизированными камерами, расположенными под углом $90^{\circ}$ по отношению друг к другу.

\section{Экспериментальные результаты}

В процессе регулировки параметров питания и условий истечения жидкости (как правило, воды) могут быть реализованы наиболее характерные типы разряда, имеющего все признаки как дугового, так и пинчевого, имеющего высоту, как правило, 4-5 mm (до 8-10 mm), с основанием на поверхности заземленного электрода менее $1 \mathrm{~mm}$ (как правило, не более $0.3 \mathrm{~mm}$ ). В $\theta$-пинчах движение электронов начинается в верхней части и имеет признаки Z-пинча, т.е. главным образом, вертикальное, а затем - вращательное в горизонтальной плоскости.

При внимательном изучении данных фото- и киносъемки обнаруживается, что верхняя часть пинча образуется в виде шарообразного облака, в отдельных случаях имеющего поверхность многогранника. В непосредственной близости от $\theta$-пинча очень часто обнаруживается наличие светящихся шарообразных и дугообразных объектов с характерным размером 2-4 mm (рис. 1).

Для изучения процессов ионизации и регистрации сопутствующих разряду излучений в непосредственной 


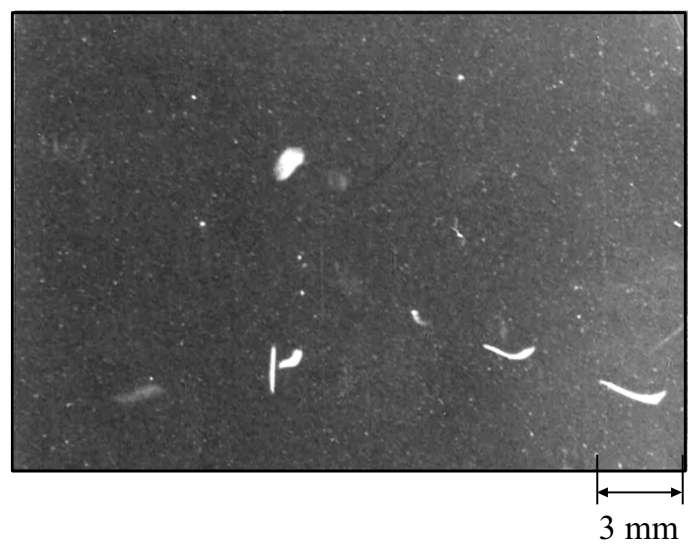

Рис. 1. Светящиеся плазмоиды шарообразного и дугообразного видов после прекращения ПРПЖ.

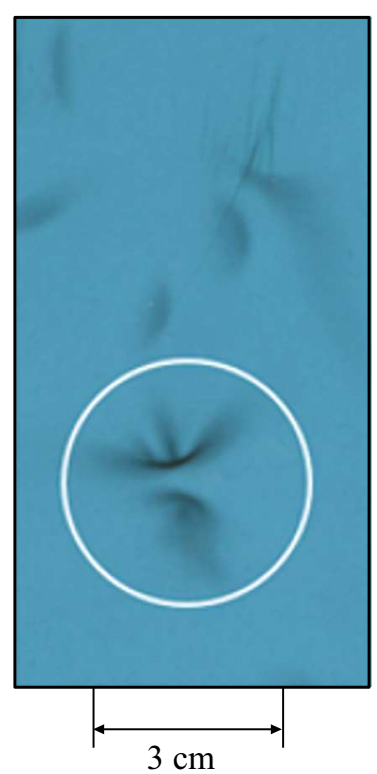

Рис. 2. Отпечатки в виде „птичек“ на рентгеновской пленке, установленной вблизи разряда.

близости от разряда (на расстоянии $\sim 20 \mathrm{~cm}$ от оси разряда) устанавливались рентгеновские пленки, плоскость которых была вертикальной, а ориентации касательной к окружности с центром на оси [6]. При изучении изображений на пленке обнаружены отпечатки, имеющие форму, близкую к эллиптической, напоминающие „Птичек с крыльями“ эллиптической формы (рис. 2). Это могло свидетельствовать о наличии в зоне локализации разряда ионизирующих излучений. Характерный поперечный размер отпечатков достигал $\sim 1 \mathrm{~cm}$, продольный - до $3 \mathrm{~cm}$.

Для выяснения причин и обстоятельств появления светящихся плазмоидов в пространстве, окружающем зону разряда, производилась киносъемка установки с применением соответствующих светофильтров и без них. Анализ результатов позволил сделать вывод о на- личии светящихся плазмоидов диаметром до нескольких $(2-5) \mathrm{cm}$, движущихся от места разряда в направлении, близком к горизонтальному, причем свечение плазмоида меняет цвет от белого до синего (голубого), а затем - красного, что может характеризоваться как „охлаждение“ плазменного сгустка в воздухе. Направление движения может определяться направлением силовых линий электрического поля, если предположить, что сгусток в целом заряжен так же, как и высоковольтный электрод (в данном случае отрицательно) [7].

Изучение поверхности коллектора, представляющего собой диэлектрическую или металлическую пластину диаметром 10-20 cm, установленную в вертикальной плоскости перпендикулярно траектории полета плазмоида на расстояниях от 5 до $50 \mathrm{~cm}$, показало следующее. Непосредственно после сеанса общей длительностью $\sim 8-10 \mathrm{~min}$ на ее поверхности, находящейся на расстоянии $\sim 50 \mathrm{~cm}$ (и более), а также через несколько десятков минут, достаточных для испарения капель воды, если расстояние менее $30 \mathrm{~cm}$, обнаруживается наличие треков, ранее там отсутствующих.

С течением времени (до нескольких суток) количество треков на поверхности коллекторов, находящихся в специальных контейнерах (чашки Петри и др.), увеличивалось, а затем оставалось без изменения. Аналогичная ситуация наблюдалась и с поверхностями металлических электродов, используемых в качестве заземленных электродов в разряде, но не подверженных непосредственному действию разрядов (на расстоянии до нескольких сантиметров от каверн, образуемых разрядом [2]).

Для установления причин появления треков было установлено круглосуточное наблюдение за поверхностями, подвергнутыми воздействию ПРПЖ полностью или частично. Видеосъемка показала наличие светящихся объектов, перемещающихся по поверхности и

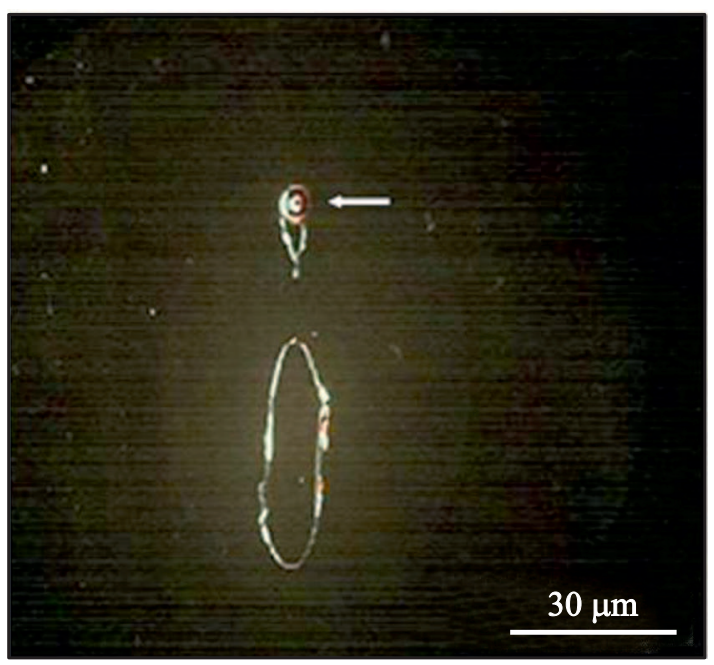

Рис. 3. Отпечаток на металлической поверхности, оставленный предположительно плазмоидами шарообразного вида и эллипсоидального сечения. 
оставляющих следы на поверхности $[8,9]$. В ночное время обнаруживаются светящиеся точки на поверхности (размер менее $1 \mathrm{~mm}$ ), обладающие светимостью до нескольких суток и более [4]. Отдельные светящиеся объекты перемещаются по поверхности. Они имеют, предположительно, торообразное строение и вращаются вокруг своей оси. В поперечном направлении они имеют размер до $50 \mu \mathrm{m}$, скорость перемещения от $1 \mathrm{~mm} / \mathrm{s}$ до $10 \mathrm{~m} / \mathrm{s}$. Цвет свечения - зеленый. В некоторых случаях внутри них наблюдаются темные зоны, предположительно вращающиеся вместе вокруг его оси тороида [7]. Светящиеся шаровидные объекты, перемещающиеся по поверхности, наблюдались и ранее (рис. 3) [4], однако их размер достигал нескольких миллиметров, а время существования не превышало нескольких секунд (микроплазмоиды торообразного вида наблюдаются в течение нескольких десятков секунд).

\section{Обсуждение результатов}

Построение модели изучаемых явлений и интерпретацию результатов экспериментов можно обосновать, основываясь на аналитических данных других экспериментов, в частности, на анализе рентгенологических исследований, практически полностью совпадающих с результатами, полученными при изучении ПРПЖ. Имеются в виду так называемые „птички“ на рентгеновских пленках, имеющие специфическую форму и размеры, практически совпадающие или близкие к описанным выше. Эти результаты получены сотрудниками НИЯУ МИФИ на ускорителях электронов, генерирующих ионизирующее тормозное излучение с максимальной энергией фотонов разного диапазона: 3-9, 20 и $\sim 320 \mathrm{MeV}$ (первые два изготовлены НИЯУ МИФИ, последний - синхротрон „Пахра“ в ФИАНе) [6]. Подобного рода отпечатки зафиксированы и другими исследователями на других установках [10]. Общим фактором, ответственным за их появление, во всех случаях является наличие ионизирующего излучения различной энергии, причем это может быть как фотонное, так и корпускулярное (электронное) излучение.

Как отмечалось в работе [6], уникальная форма отпечатка может свидетельствовать о наличии на поверхности рентгеновской пленки (или в непосредственной близости от нее) объекта, содержащего ионизирующие частицы каскадного процесса, в частности, электроны ионизованного воздуха, образующие поток, имеющий в сечении форму отпечатка на пленке. Восстановление двумерного изображения при переходе к объемному (трехмерному) объекту указывает на допустимость предположения об образовании в воздухе объектов, по форме напоминающих $\theta$-пинч над поверхностью заземленного электрода. Присутствие последнего в воздухе может найти объяснение в случае, если допустить существование в ионизованной воздушной среде объектов, обладающими свойствами магнитного монополя [11].
Главным отличием от известных моделей, объясняющих существование монополей с электронным „шлейфом“ в виде потока вращающихся электронов [11,12], является количественная характеристика (размеры, количество электронов и др.), сопровождаемая качественными отличиями (стабильность во времени, функциональное воздействие на среду и др.).

Объяснение описываемым явлениям с позиций физики плазмы найдено с привлечением результатов общей теории относительности (ОТО), полученных при анализе механизмов формирования пинчевых плазмоидов (без магнитных монополей) $[13,14]$.

Для обобщения данных результатов на случай наличия в объеме ионизации магнитных монополей можно сделать предположение о существовании в данном объеме (до ионизации) диполей, в целом имеющих до ионизации нулевой магнитный заряд, но после воздействия ионизирующего излучения образующих частицы разноименной полярности („северный“ и „южный“). На эту роль могли бы претендовать молекулы воздуха и водяного пара $\left(\mathrm{H}_{2}, \mathrm{O}_{2}, \mathrm{~N}_{2}\right.$ и др.). В случае если в числе данных молекул содержатся (по крайней мере, после воздействия ионизирующего излучения) такие, которые имеют монополи типа протонов с электронами или без них (электроны осуществляют до воздействия ионизирующего излучения молекулярную связь), после распада могут образовывать стабильные частицы типа дионов, рассмотренных Дираком и его современниками [12]. Дионы могут иметь как электрический заряд, так и магнитный.

Было показано [12], что условие квантования Дирака обобщается на существование и взаимодействие двух таких частиц. Показано, что только предположение о существовании частиц, несущих как и магнитный, так и электрический заряды, может обеспечить решение проблемы дуальной инвариантности электродинамики.

Следующим важным этапом многокаскадного процесса предположительно является образование пинчевых плазмоидов, содержащих магнитные заряды (и, вероятнее всего, именно дионы, образованные после ионизации и разделения их внешним полем).

Существование в квазистабильном состоянии новообразований кластерного типа, содержащих положительно и отрицательно заряженные частицы, а также нейтральные атомы и молекулы, как сказано ранее, возможно как теоретически, так и экспериментально $[13,14]$.

Для оценки параметров пинчевых плазмоидов, содержащих также магнитные заряды на основе дионов типа „монополь-протон“ (а именно протоны рассматривались в данном качестве [12]), можно предположить, что при формировании плазмоида под влиянием электрического поля в межэлектродном пространстве происходит разделение дионов в соответствии с полярностью, т.е. новообразованный плазмоид состоит из дионов одного вида, например, типа „монополь-протон“. Расталкивание таких дионов в основном благодаря магнитному полю магнитных зарядов может быть скомпенсировано 
совокупным действием гравитационного поля и кулоновского (электрического) поля за счет избыточного (по сравнению со случаем без магнитных зарядов) заряда электронов внутри плазмоида. При этом можно считать, что расталкивание за счет магнитных сил эквивалентно расталкиванию одноименных элементарных зарядов с учетом того, что магнитный заряд связан с элементарным электрическим зарядом соотношением [12]

$$
\frac{2 \mu e}{c}=n \hbar
$$

где $n-$ произвольное целое число, $\hbar-$ постоянная Планка (минимальное значение $\mu$ в 68.5 раз больше $e$ ).

Предварительные оценки показывают, что при атмосферном давлении $1 \cdot 10^{5} \mathrm{~Pa}$ и при максимальной степени ионизации, обеспечиваемой ионизирующим излучением, в воздухе может образовываться ионизованная среда со средней плотностью электронов и ионов на уровне $10^{18}-10^{19} \mathrm{~cm}^{-3}$. Можно в первом приближении считать плотность дионов не более $\sim 10^{-2}$ от уровня $3 \cdot 10^{19} \mathrm{~cm}^{-3}$, т.е. порядка $10^{17} \mathrm{~cm}^{-3}$ (будет показано далее, что более реальная оценка не $10^{-2}$, a $\sim 10^{-4}$ ).

Если в качестве примера рассматривать типичный вариант, когда первоначально сформированный пинчевой плазмоид имеет объем $\sim 0.1 \mathrm{~cm}^{3}$, то после компрессии в процессе пинчевания над поверхностью он имеет объем порядка $10^{-3} \mathrm{~cm}^{3}$, т.е. в 100 раз меньше. Плотность электронов в этом случае близка к величине именно $10^{17} \mathrm{~cm}^{-3}$.

В плоскости нижнего электрода в зоне основания пинча образуется колоссальная плотность энерговыделения, о чем свидетельствуют материаловедческие исследования [15]. Если считать, что импульсный ток в разряде составляет хотя бы $1 \mathrm{kA}$, а напряжение разряда достигает $10 \mathrm{kV}$, то импульсная плотность мощности на электроде составит

$$
P=U \cdot \frac{I}{S} \approx \frac{4 \cdot 10^{7} \mathrm{~W}}{\pi \cdot(0.1)^{2} \mathrm{~cm}^{2}} \approx 1.3 \cdot 10^{9}\left[\mathrm{~W} / \mathrm{cm}^{2}\right]
$$

При этих значениях происходит мгновенное расплавление и частичное испарение металла электрода в зоне диаметром $100 \mu \mathrm{m}$ с глубиной $\sim 100 \mu \mathrm{m}$ (и более), с последующим быстрым охлаждением. В этой зоне и над ней образуется облако пара, состоящего из молекул и атомов металла, который подвергается ионизации электронным потоком. Плотность ионов и электронов в этой зоне, естественно, меньше, чем плотность в твердом теле $\left(10^{23}-10^{24} \mathrm{~cm}^{-3}\right)$, но выше, чем в атмосферном воздухе даже после компрессии и по предварительным оценкам она может быть порядка $5 \cdot 10^{19} \mathrm{~cm}^{-3}$. При выборе этого параметра учитывались результаты наблюдений за динамикой плазмоидов после разряда, свидетельствующие о близости значений их плотности к плотности атмосферы (нет резких подъемов и падений во время движения).
Как показано в работах [13,14], устойчивое существования плазмоида без магнитных зарядов теоретически возможно при следующих параметрах: количество протонов $N_{p} \approx 2 \cdot 10^{14}$; количество электронов $N_{e}=$ $=9.25 \cdot 10^{13}$; плотность числа зарядов $n_{p}=5 \cdot 10^{19} \mathrm{~cm}^{-3}$; радиус плазмоида в основании пинча $R_{n}=100 \mu \mathrm{m}$. При этом масса плазмоида составит около $7 \cdot 10^{-10} \mathrm{~g}$. Расчеты допускают и несколько иную комбинацию параметров: радиус плазмоида $50 \mu \mathrm{m}$, число протонов $N_{p}-$ $10^{16}$, число электронов - $10^{16}$, число нескомпенсированных зарядов $N_{a} \approx 10^{15}$, масса плазмоида $-10^{-8} \mathrm{~g}$.

Если предположить, что нескомпенсированный заряд в плазмоиде перекомпенсирует заряд дионов (до 100 раз, см. уравнение (1)), то можно оценить количество последних величиной порядка $N_{d} \approx 10^{13}$. Учитывая, что квант магнитного потока составляет величину $\sim 2 \cdot 10^{-15} \mathrm{~Wb}$, общий магнитный поток всех магнитных зарядов составит $\sim 2 \mathrm{~Wb}=2 \mathrm{~T} \cdot \mathrm{m}^{2}$. Это значение представляется значительно завышенным, если судить по результатам исследований динамики электронов в магнитном поле таких кластеров, находящихся предположительно в поверхностном слое после окончания пинчевого разряда, либо в воздухе до их перемещения на ближайшие объекты (рентгеновскую пленку, диэлектрические или металлические пластины и др.).

Первоначально присутствие таких долгоживущих образований в металлической подложке обнаруживалось в процессе измерений с помощью электронного микроскопа, когда изображение во вторичных электронах исчезало при наведении зондирующего первичного электронного пучка на точечные источники, наблюдаемые в оптический микроскоп и отсутствующие до помещения их в зону разряда. Треки на поверхности обнаруживались в послеразрядный период как следы перемещения пинчевых плазмоидов. Гораздо более низкое значение, приближенное к реальному, может быть получено на основании анализа отпечатков в виде „птичек“ или близких к ним по форме (рис. 2) в предположении, что электронный „шлейф“, являющийся дополнением поля кластера, содержащего магнитный заряд, образован куперовскими парами, обладающими повышенной проникающей способностью [16] (для доказательства этого планируется проведение дополнительных исследований). Происхождение этих электронов может объясняться наличием ионизирующего излучения, ответственного за появление в зоне локализации разряда магнитных зарядов. Энергетический спектр этих электронов определяется, как известно [17], величиной энергии ионизации и энергией ионизирующих частиц (фотонов, электронов).

Косвенным доказательством этого является то, что размер „крыльев птичек“, полученных на синхротроне с энергией фотонов ионизирующего излучения в несколько раз больше, чем, например, на линейном ускорителе электронов на энергию 2-4 MeV. Движение электронов в составе куперовских пар в атмосфере и в вакууме может носить близкий характер и оцениваться по формуле 


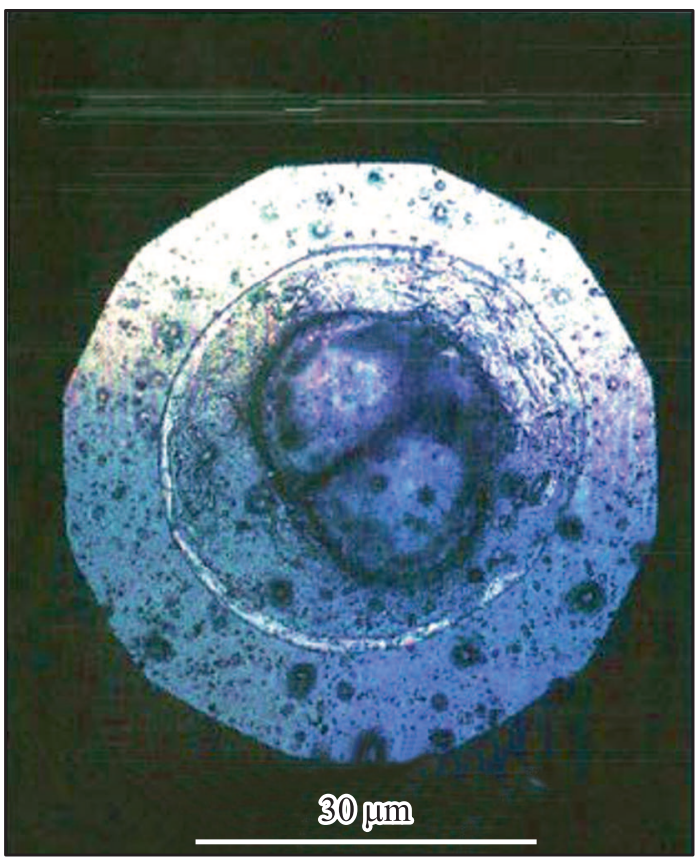

Рис. 4. Отпечаток на диэлектрической пластине, имеющий форму „крыльев бабочки“.

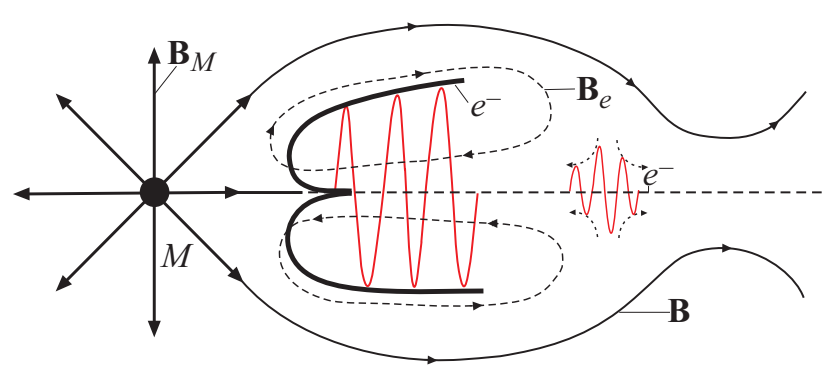

Pис. 5. Схема формирования плазмоидов в зоне локализации магнитного заряда с шлейфом электронов. $\mathbf{B}_{e}-$ силовая линия магнитного поля электронного потока; $\mathbf{B}=\mathbf{B}_{e}+\mathbf{B}_{M} ; \mathbf{B}_{M}-$ силовая линия магнитного поля магнитного заряда.

для „пробочного“ соотношения в так называемом „пробкотроне“, имеющем конфигурацию магнитного поля, близкую к магнитному полю монополя (в данном случае магнитного поля кластера, содержащего магнитные заряды), и электрическом поле коллектива протонов, в частности, образующих дионы.

Для анализа свойств магнитного кластера на основе дионов в составе плазмоида, сопровождаемого „шлейфом“ из электронов, обладающих свойствами „куперовских пар“, могут быть использованы следы такого рода объектов на диэлектрических или металлических поверхностях, находящихся в зоне непосредственного разряда или вблизи от него на расстоянии нескольких сантиметров. В некоторых случаях они имеют в сечении характер несимметричного эллипсоида (рис. 3), в других случаях сечение объекта, идентифицируемого как „шлейф“, подобно „крыльям бабочки“ (рис. 4). Если в первом случае можно отнести „шлейф“ эллипсоидального типа к тому же классу, что и „крылья птичек“, то во втором случае конфигурация электронной оболочки позволяет провести аналогию с радиационным поясом Земли в магнитном поле дипольного типа [6]. В этом случае можно полагать, что в совокупности с магнитным полем кластера магнитных зарядов (дионов) электронный шлейф образует объект, содержащий оболочку электронов, совершающих движение по магнитной поверхности, наблюдаемую извне по свечению плазмоида (рис. 5). Движение электронов носит характер ,замагниченных“ электронов в совокупном магнитном поле, причем одни и те же электроны совершают вращение вокруг общей оси и градиентный дрейф как по внешней, так и по внутренней (вблизи оси) части магнитной поверхности (предположительно, кластер магнитных зарядов образован частицами, имеющими положительный электрический заряд).

Динамика электронов в поле магнитного заряда подробно изучена в работах $[11,12]$. Движение электронов вблизи оси связано с замедлением электронов в воздухе

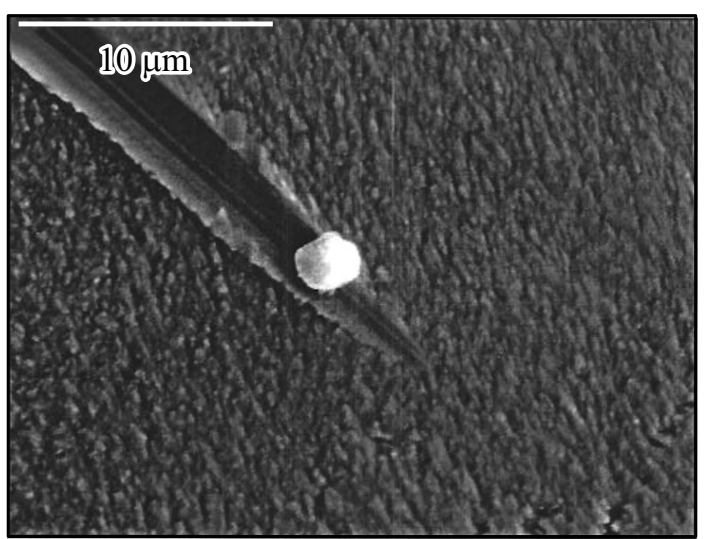

Рис. 6. Следы („траншеи“) на медной пластине после окончания сеанса с ПРПЖ. В конце „траншеи“ - „комок“ из такого же вещества.

\section{$20 \mu \mathrm{m}$}

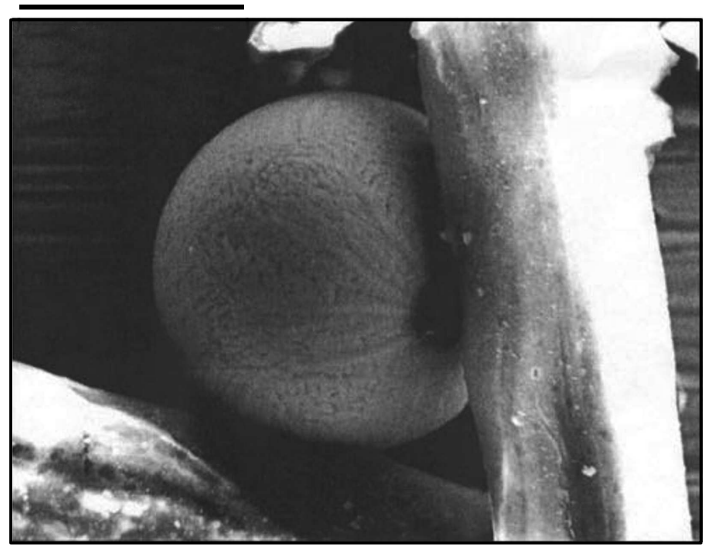

Рис. 7. Сферическое новообразование и нить на поверхности лезвия после ПРПЖ вблизи от подложки. 


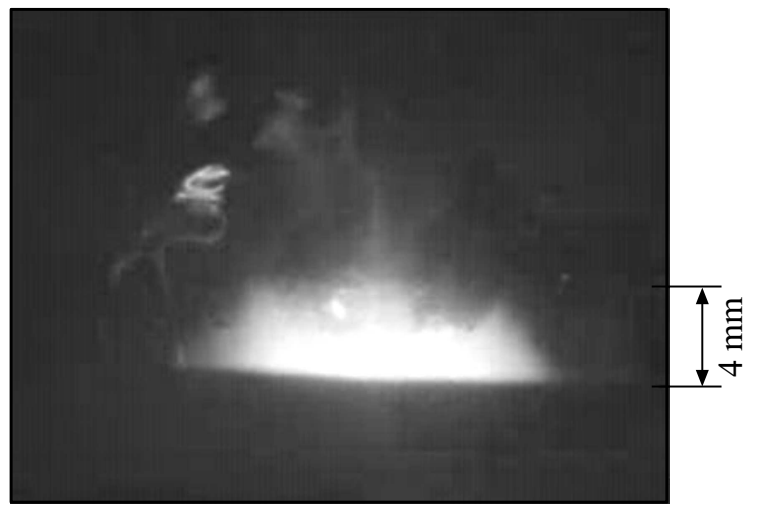

Рис. 8. Светящаяся нить „скругленного“ вида в зоне ПРПЖ (,долгоживущая“).

и уменьшением радиуса вращения и протяженности траектории после отражения от „магнитной пробки“. В правой части рисунка можно отметить появление „каспа“, соответствующего зоне в правой части рис. 4.

Если локальная плотность электронов в оболочке (по крайней мере, на внешней магнитной поверхности) достигает величины более той, которая имеется в твердом теле $\left(\sim 10^{24} \mathrm{~cm}^{-3}\right)$ (при средней плотности по всему объему тороида, образованной компонентами кластера, на уровне указанных выше примеров), то можно объяснить появление треков на поверхности, по которой перемещается кластер, а также наличие внутри него атомов и молекул вещества подложки. Направление и скорость перемещения кластера должны определяться наличием внешних полей (электрического и магнитного, в том числе полей Земли), а также внутренних механизмов гидродинамического характера (реакция на взаимодействие электронов оболочки с веществом и др.). Характерные следы взаимодействия такого рода объектов зафиксированы в большом количестве (рис. 6).

По окончании процесса „выедания“ атомов вещества происходит предположительно остывание и конденсация вещества в виде шаровидных объектов, имеющих структуру, напоминающую кожуру апельсина (рис. 7) во многих случаях с отверстием на оси, уходящим вглубь. На фотографии изображены сразу два объекта, обнаруженные на подложке в послеразрядный период шарик описанного типа и нить, имеющая те же поперечные размеры, что и шарик. Длина такого рода „нитей“ может составлять несколько сантиметров. Они, как правило, состоят из того же вещества, что и подложка, и кислорода. В отдельных случаях они содержат углерод (присутствие последнего может объясняться распылением графитового электрода - трубки подачи жидкости).

Происхождение этих нитей можно связать с теми же процессами, что и шариков, а именно образованием плазменных нитей в процессе разряда, с последующей концентрацией образовавшихся паров испаренного металла высоковольтных электродов (металл, графит). Линейные плазмоиды имеют один или два светящихся конца (в первом случае второй конец уходит в поверхность). В некоторых случаях светящиеся нити существуют в течение нескольких секунд, незначительно меняя форму (рис. 8).

Теоретическое обоснование возможности существования стабильного образования данного типа было дано в работах $[13,14]$. О наличии магнитных полюсов может свидетельствовать свечение концов.

В послеразрядный период появление таких нитей могло бы рассматриваться аналогично появлению „шариков“", а именно активного кластера с выходом на поверхность в качестве плазмоида с последующим перемещением по поверхности и „выеданием“ атомов вещества подложки. В конце трека данное вещество в этом случае формируется не в виде шарика, а в виде нити или в виде нити с шариком на конце (рис. 9).

Специфическая форма „траншей“ или „треков“ после перемещения плазмоида по поверхности металла в отдельных случаях практически совпадает с „траншеями“ после перемещения шаровой молнии по поверхности металлической конструкции (рис. 10), что указывает на возможность обобщения полученных в лаборатории результатов на природные явления.

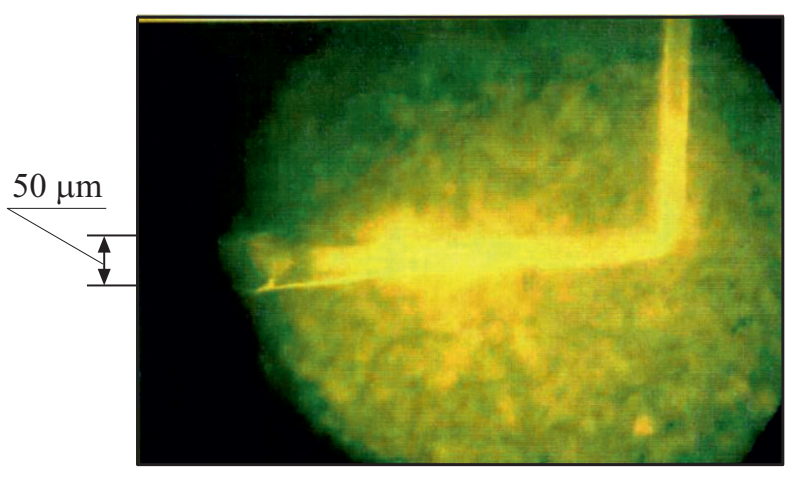

Рис. 9. Нить с шариком на конце.

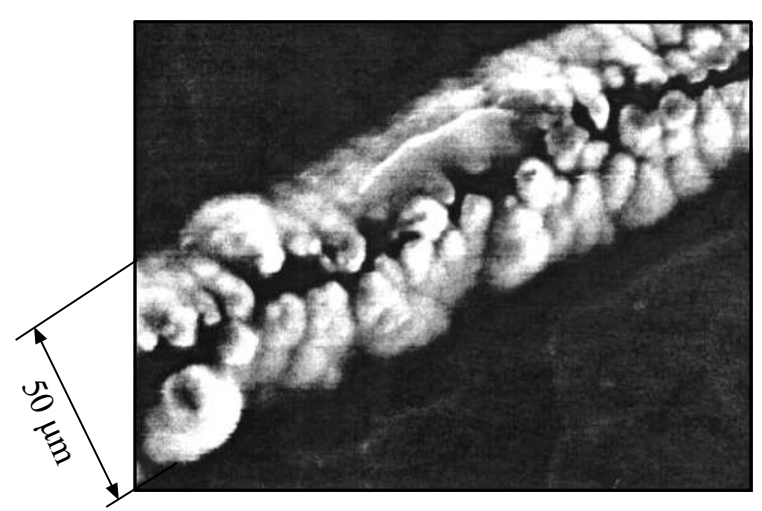

Рис. 10. „Траншея“ на образце из меди, подвергнутом воздействию ПРПЖ и имеющая формы „гусеницы“. Состав - медь, кислород. 


\section{Заключение}

Обобщающий анализ экспериментальных результатов, полученных в процессе исследования сильноточных пинчевых и дуговых разрядов, происходящих в импульсном режиме на поверхности струи воды и в окружающем пространстве при напряжении на электродах в несколько киловольт, позволил построить феноменологическую модель многокаскадного процесса, в том числе в послеразрядный период. Установлен факт появления в окружающем пространстве долгоживущих плазмоидов различного типа, имеющих шаровидную, ниточную и другие конфигурации. Особый интерес вызывает появление на поверхности объектов, подвергнутых воздействию разряда, светящихся плазмоидов, способных перемещаться, оставляя царапины. Их присутствие обнаруживается как в процессе сеанса между импульсами разряда, так и через значительные промежутки времени. Светящиеся плазмоиды способны перемещаться в воздухе на расстояния до нескольких десятков сантиметров от разряда. Металлические и диэлектрические поверхности покрываются значительным количеством треков специфической конфигурации и различной формы. Построение модели плазмоида на основе электронов, ионов, нейтральных атомов и молекул позволяет обосновать существование стабильного состояния при определенном значении параметров (плотности и числа зарядов, размеров и масс плазмоидов и др.). В основу теории ранее была положена гравитационная модель общей теории относительности. В настоящей работе высказано предположение о том, что решающим фактором, ответственным за всю совокупность описываемых явлений и процессов, является наличие в объеме ионизации незначительного по количеству, но весьма важного компонента, а именно магнитных зарядов. Образование зарядов может быть связано с ионизацией первоначально нейтральных по заряду (электрическому и магнитному) молекул, например, водорода, которые после ионизации образуют пары так называемых дионов, теоретически предсказанных почти 100 лет назад. После разделения во внешних полях этих компонент в зонах локализации плазмоидов при достаточной концентрации возможно появление плазмоидов с уникальными свойствами, в частности, присущими элементарным магнитным зарядам, а именно формирование электронного „шлейфа“ макроразмерного характера. Свидетельства появления таких „шлейфов“ имеются среди данных рентгенологического анализа не только в разрядах по струе воды, но и на рентгеновских пленках в окрестности зоны ионизации воздуха тормозным излучением ускорителей разной энергии (от единиц до сотен $\mathrm{MeV}$ ).

Одним из аргументов в пользу такой модели является наличие светящихся плазмоидов эллипсоидального сечения и предположительно тороидальной формы, вращающихся вокруг своей оси, о чем свидетельствуют перемещения непрозрачной части тороида (вид сверху из объектива микроскопа). Образование „траншеи“ после прохождения объекта логичнее связать с выеданием атомов подложки тороидальной оболочкой, предположительно образованной электронами, совершающими инфинитное движение в магнитном поле диполя, образованного магнитными зарядами совместно с электронным „шлейфом“. Динамика электрона в поле монополя изучалась в свое время Дираком, предсказавшим позитрон и монополь и теоретически исследовавшим их свойства.

Вещественным доказательством в обосновании данной гипотезы могут явиться шаровидные объекты с отверстием на оси, обнаруживаемые в значительном количестве в послеразрядный период на поверхностях, покрытых треками („траншеями“). Они рассматриваются как „остывшие“ плазмоиды с осью симметрии, совпадающей с осью вращения. Нити из вещества подложки, покрытые кислородно-углеродной оболочкой, могут быть отнесены к объектам того же типа. Их существование, в частности, объясняется фиксацией на кинопленке светящихся нитей в течение разряда, а также между разрядными импульсами в течение нескольких секунд.

Полученные результаты могут быть полезны при описании таких природных явлений, как грозы и смерчи, поскольку условия протекания процессов и результаты могут иметь общую природу и последствия (шаровая молния и др.).

Особую актуальность имеет вопрос обеспечения безопасности для окружающих технологического процесса и потребителей продукции, полученной с его использованием. В последнем случае речь может идти о прямом контакте обработанной поверхности с кожными покровами человека. В случае, если процесс обработки ПРПЖ не является финальной частью процесса, он не представляет опасности, так как поверхностный слой снимается при шлифовке и полировке, а также деформационном упрочнении со шлифовкой, и поступает в отходы, где влияние наличия магнитных зарядов практически пренебрежимо мало. В случае наличия магнитных зарядов в воздухе за счет принудительной вентиляции, необходимой для снижения концентрации продуктов электролиза воды до необходимого уровня (опасность образования гремучего газа), концентрации магнитных зарядов мизерна.

Однако полученные в результате аналогичных процессов в смежных областях новообразования в виде „Птичек“, например, на ускорителях, могут иметь важное значение, поскольку влияние этих объектов в случае больших концентраций в воздухе (а именно так и обстоит дело на ускорителях) абсолютно не изучено и требует дополнительных исследований.

\section{Финансирование работы}

Работа выполнена в рамках Соглашения № 17-7220228 между Российским научным фондом и Национальным исследовательским ядерным университетом „МИФИ“ о предоставлении гранта на проведение фундаментальных и поисковых научных исследований. 


\section{Список литературы}

[1] Богданович Б.Ю., Калин Б.А., Нестерович А.В., Пучков А.Н. Периодический дуговой разряд в потоке жидкости как средство повышения микротвердости и „травления“ поверхности металла. Тезисы IV Всероссийской конференции по модификации свойств конструкционных материалов пучками заряженных частиц. Томск: ТПУ, 1996. C. 102.

[2] Нестерович А.В., Богданович Б.Ю., Калин Б.А., Волков Н.В., Лубков В.М. Упрочнение поверхности углеродистой стали при воздействии периодического высоковольтного разряда в потоке жидкости. Сборник научных трудов „Научная сессия МИФИ-99“. М.: МИФИ, 1999. T. 5. C. $20-22$.

[3] Нестерович А.В., Богданович Б.Ю., Калин Б.А., Волков Н.В., Мизин Ю.В. Модификация приповерхностного слоя материалов в области воздействия высоковольтного разряда в потоке жидкости. Сборник научных трудов „Научная сессия МИФИ-2000“. М.: МИФИ, 2000. Т. 9. C. 34-35.

[4] Богданович Б.Ю., Волков Н.В., Косточко Ю.П., Лень Н.А., Нестерович А.В., Старостин А.И. Экспериментальное исследование квазипериодического импульсного разряда, инициируемого в потоке жидкости и приэлектродном пространстве. Инженерная физика. М.: МИФИ, 2000. № 1. C. 19-23.

[5] Bogdanovich B.Y., Kalin B.A., Nesterovich A.V. // Appl. Res. 2016. Vol. 7. N 5. P. 673-681.

[6] Нестерович А.В., Богданович Б.Ю., Буянов Г.О. Экспериментальное исследование ионизации окружающей среды в зоне периодического разряда в потоке жидкости (ПРПЖ). IV Международная конференция „Лазерные, плазменные исследования и технологии“ Лаплаз-2018. Сборник научных трудов. М.: НИЯУ МИФИ, 2018. 543 с.

[7] Богданович Б.Ю., Волков Н.В., Лень Н.А., Нестерович А.В. // ЖТФ. 2019. Т. 89. Вып. 4. С. 507-511.

[8] Bogdsnovich B.Yu., Nesterovich A.V. Periodic discharge in a stream of liquid and anomalous phenomenas in the discharge period. $11^{\text {th }}$ International Workshop on Magneto-Plasma aerodynamics (abstracts) Moscow. 10-12 April. 2012. P. 108.

[9] Bogdsnovich B.Yu., Nesterovich A.V. // J. Phys. Conf. Series. 2016. Vol. 747. UNSP 012001.

[10] Жигалов В.А. // Журнал формирующихся направлений науки (ЖФНН). 2015. № 9(3). С. 55-62

[11] Вишневский Р.П. Сверхпроводимость монополя Дирака. Электронный ресурс: режим доступа: http://lightdynamics.narod.ru/Sverhprovodimost_monopolya_Dirka.htm. Дата обращения: 10.09.2018.

[12] Сборник статей „Монополь Дирака“ / Под ред. Б.М. Болотовского, Ю.Д. Усачева. М.: Мир, 1970. С. 65.

[13] Богданович Б.Ю., Нестерович А.В., Суханова Л.А., Хлестков Ю.А. // Изв. вузов. Физика. 2016. Т. 59. № 10. C. 109-119.

[14] Богданович Б.Ю., Нестерович А.В., Суханова Л.А., Хлестков Ю.А. Оценка параметров пинчевых плазмоидов на основе гравитационной модели. Фундаментальные и прикладные исследования в современном мире. Материалы Международной научно-практической конференции. 2014. T. 1. C. 11-15.
[15] Нестерович А.В., Фетисов Г.П. // Инженерная физика. 2007. № 5. C. 7-12.

[16] Авраменко Р.Ф. Будущее открывается квантовым ключом. М.: Химия, 2000. 352 с.

[17] Большая Советская Энциклопедия, в 30 т. М.: Советская энциклопедия, 1972. Т. 20. С. 372. 